\title{
The Electromagnetic Properties of the Light Pseudoscalar Mesons via the Primakoff Effect
}

\author{
D. S. Dale \\ University of Kentucky, Department of Physics and Astronomy, Lexington, KY 40506, USA
}

(for the PrimEx collaboration)

Received on 3 November, 2003

\begin{abstract}
The PrimEx (Primakoff Experiment) Collaboration is currently preparing to perform a high precision (1.4\%) measurement of the two photon decay width of the neutral pion, $\Gamma_{\pi^{o} \rightarrow \gamma \gamma}$. The proposed $12 \mathrm{GeV}$ upgrade of the CEBAF accelerator will enable a significant enlarging of the scope of these studies to include measurements of the two photon decay widths of the $\eta$ and $\eta^{\prime}$ mesons, as well as of the transition form factors of all the light pseudoscalar mesons $\left(\pi^{o}, \eta, \eta^{\prime}\right)$. These precision measurements would have a significant impact on the experimental determination of the ratios of the light quark masses $\left(m_{u}, m_{d}, m_{s}\right)$, and on our understanding of some fundamental issues in QCD.
\end{abstract}

\section{The Radiative Widths}

The two-photon decay mode of the $\pi^{0}$ involves the explicit breaking of a classical symmetry by the quantum fluctuations of the quark fields coupling to a gauge field[1]. This phenomenon, called anomalous symmetry breaking, is of pure quantum mechanical origin, and in this case involves the coupling of the quarks to photons[2]. In the limit of vanishing quark masses, the anomaly leads to the $\pi^{0} \rightarrow \gamma \gamma$ decay width $[1,2]$ :

$$
\Gamma=\frac{M_{\pi}^{3} \alpha_{e m}^{2}}{64 \pi^{3} F_{\pi}^{2}}=7.725 \pm 0.044 e V
$$

where $F_{\pi}=92.42 \pm 0.25 \mathrm{MeV}$ is the pion decay constant[3]. The current experimental value, summarized in figure 1, is $7.84 \pm 0.56 \mathrm{eV}[3]$ and is in good agreement with the predicted value in the chiral limit. Stimulated by the PrimEx proposal, next to leading order calculations have been performed giving $\Gamma_{\pi^{0} \rightarrow \gamma \gamma}=8.10 \mathrm{eV}$ with an estimated uncertainty of less than $1 \%$ [4][5]. With its $1.4 \%$ level of precision, such effects will be tested in the PrimEx experiment.

The availability of $12 \mathrm{GeV}$ beams at Jefferson Lab will also enable measurements of the $\eta$ radiative width. One decay that is particularly important is the decay $\eta \rightarrow \pi \pi \pi$, which is driven by isospin breaking, and thus gives access to the quark mass ratio $\left(m_{u}-m_{d}\right) / m_{s}$. In a very elegant analysis, Leutwyler[11] constructed a relation, now called Leutwyler's ellipse, given by:

$$
\left(\frac{m_{u}}{m_{d}}\right)^{2}+\frac{1}{\mathcal{Q}^{2}}\left(\frac{m_{s}}{m_{d}}\right)^{2}=1
$$

where the semi-major axis $Q$ is given by the ratio:

$$
\mathcal{Q}^{2}=\frac{m_{s}^{2}-\hat{m}^{2}}{m_{d}^{2}-m_{u}^{2}}
$$

with $\hat{m}=\left(m_{u}+m_{d}\right) / 2$. One way to determine $\mathcal{Q}$ is given by a ratio of meson masses:

$$
\mathcal{Q}^{2}=\frac{M_{K}^{2}}{M_{\pi}^{2}} \frac{M_{K}^{2}-M_{\pi}^{2}}{\left(M_{K^{0}}^{2}-M_{K^{+}}^{2}\right)_{Q C D}}\left[1+O\left(m_{\text {quark }}^{2}\right)\right]
$$

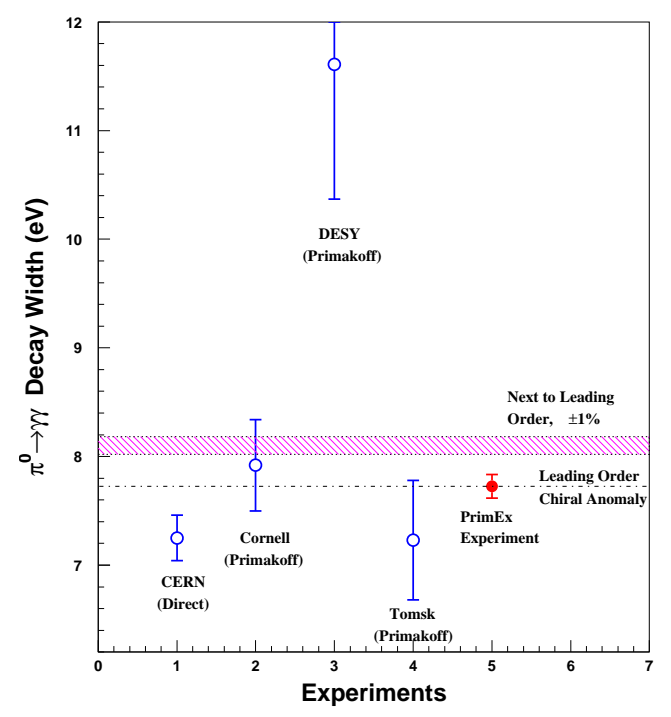

Figure 1. $\pi^{o} \rightarrow \gamma \gamma$ decay width in eV. The dashed horizontal line is the leading order prediction of the axial anomaly (equation $1)[1,2]$. The shaded band is the recent next-to-leading order prediction. The experimental results with errors are for : (1) the direct method[7]; $(2,3,4)$ the Primakoff method [8, 9, 10]; (5) the expected error for the PrimEx experiment, arbitrarily plotted to agree with the leading order prediction. 
The chief problem in extracting $\mathcal{Q}$ from this relation arises from the uncertainties in the electromagnetic contributions to the $K^{0}-K^{+}$mass difference. Another way to extract $\mathcal{Q}$ is by means of $\eta \rightarrow \pi \pi \pi$ decays which have negligibly small electromagnetic corrections due to chiral symmetry. As emphasized by Leutwyler[11], the main errors in determining $\mathcal{Q}$ using $\eta \rightarrow \pi \pi \pi$ decays is due to the experimental uncertainties in the partial width $\Gamma_{\eta \rightarrow \pi \pi \pi}$, which are in turn determined by the two-photon width $\Gamma_{\eta \rightarrow \gamma \gamma}$ and branching ratio. Fig. 2 shows the significant improvement that a Primakoff $\eta$ production experiment at Jefferson Lab could make in the determination of this quark mass ratio.

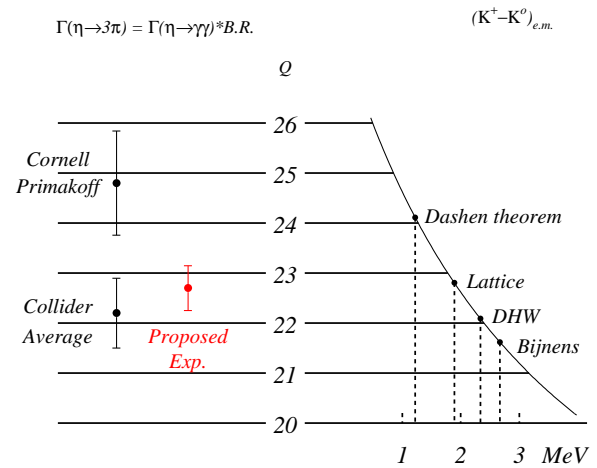

Figure 2. The importance of $\Gamma_{\eta \rightarrow \gamma \gamma}$ in the measurement of $\mathcal{Q}$. The 1.h.s. indicates the values of $\mathcal{Q}$ corresponding to the Primakoff and collider experimental results for the $\Gamma_{\eta \rightarrow \gamma \gamma}$. The r.h.s. shows the results for $\mathcal{Q}$ obtained with four different theoretical estimates for the electromagnetic self energies of the kaons. Taken from Ref. [11].

\section{The Primakoff method}

We plan to use quasi-monochromatic photons at Jefferson Lab to measure the absolute cross section of small angle pseudoscalar meson photoproduction from the Coulomb field of nuclei. The invariant mass and angle of the meson will be reconstructed by detecting the decay photons from the $P \rightarrow \gamma \gamma$ reaction, where $P$ represents the $\pi^{o}, \eta$, or $\eta^{\prime}$ meson. For unpolarized photons, the Primakoff cross section is given by[9]:

$$
\frac{d^{3} \sigma_{P}}{d \Omega}=\Gamma_{\gamma \gamma} \frac{8 \alpha Z^{2}}{m^{3}} \frac{\beta^{3} E^{4}}{Q^{4}}\left|F_{e . m .}(Q)\right|^{2} \sin ^{2} \theta_{P}
$$

where $\Gamma_{\gamma \gamma}$ is the decay width, $Z$ is the atomic number, $m$, $\beta, \theta_{P}$ are the mass, velocity and production angle of the meson, $E$ is the energy of incoming photon, $Q$ is the momentum transfer to the nucleus, and $F_{e . m .}(Q)$ is the nuclear electromagnetic form factor, corrected for final state interactions of the outgoing meson. Some care must be taken to isolate the Primakoff mechanism from competing processes. In particular, the full cross section is given by: $\frac{d^{3} \sigma}{d \Omega_{\pi}}=\frac{d \sigma_{P}}{d \Omega}+\frac{d \sigma_{C}}{d \Omega}+\frac{d \sigma_{I}}{d \Omega}+2 \cdot \sqrt{\frac{d \sigma_{P}}{d \Omega} \cdot \frac{d \sigma_{C}}{d \Omega}} \cos \left(\phi_{1}+\phi_{2}\right)$.

The nuclear coherent cross section is given by $\frac{d \sigma_{C}}{d \Omega}$, and the incoherent cross section is $\frac{d \sigma_{I}}{d \Omega}$. The relative phase between the Primakoff and nuclear coherent amplitudes without final state interactions is given by $\phi_{1}$, and the phase shift of the outgoing meson due to final state interactions is given by $\phi_{2}$.

Kinematical considerations enable one to separate the Primakoff effect from other photoproduction mechanisms. (See Fig. 3.) We are currently constructing a detector with good angular resolution to characterize nuclear coherent production, and good energy resolution in the decay photon detection will enable an invariant mass cut to suppress multi-photon backgrounds.

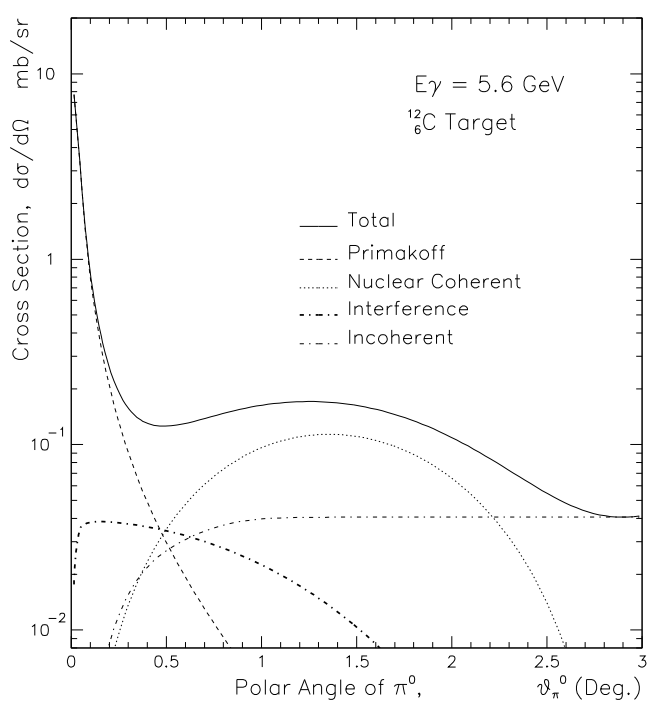

Figure 3. Angular behavior of the electromagnetic and nuclear $\pi^{o}$ photoproduction cross sections for ${ }^{12} \mathrm{C}$ in the $6.0 \mathrm{GeV}$ energy range.

\section{Transition form factor measure- ments}

Using Primakoff electroproduction it is also possible to measure the transition form factors $F_{\gamma \gamma^{*} P}$ for one off shell photon. So far, the transition form factors have been determined in collider experiments[12] with relatively large $Q^{2}$ $\left(Q^{2} \geq 0.6 \mathrm{GeV}^{2}\right)$, except for the recent measurement for the $\eta^{\prime}$ measured by the L3 collaboration[13] where the momentum transfer is as low as $0.05 \mathrm{GeV}^{2}$ but with big error on $Q^{2}$. Measurements of the $\pi^{0}, \eta$ and $\eta^{\prime}$ transition form factors at very low $Q^{2}\left(\sim 0.001-0.5 \mathrm{GeV}^{2}\right)$ are particularly important in the extraction of the slope of the transition form factor, and to measure the size of the meson's electromagnetic interaction radius model independently. Currently, there is no 
first principles theoretical determination of the form factors. In ChPT there are two sources of contributions [14], one is the long distance contribution from meson loops, and the other is a counterterm or short distance contribution. ChPT pins down the first, and for the second a model is needed. The long distance contributions are small, as they only provide a small fraction of the fall off of the form factor. A determination of the slope of the $\pi^{0}$ and $\eta$ form factors would allow one to uniquely fix a low energy constant $\mathcal{O}\left(p^{6}\right)$ in the effective chiral Lagrangian[5][14]. In addition, one important reason to better understand the transition form factors of the $\pi^{0}, \eta$ and $\eta^{\prime}$ is that pseudoscalar exchange is the major contribution to the hadronic light-by-light scattering part of the muon anomalous magnetic moment[15] and is thus clearly crucial for measurements of $a_{\mu}$ that search for "new physics" beyond the Standard Model. Expected results on the $\pi^{o}$ using $12 \mathrm{GeV}$ beams at Jefferson Lab are shown in figure 3 .

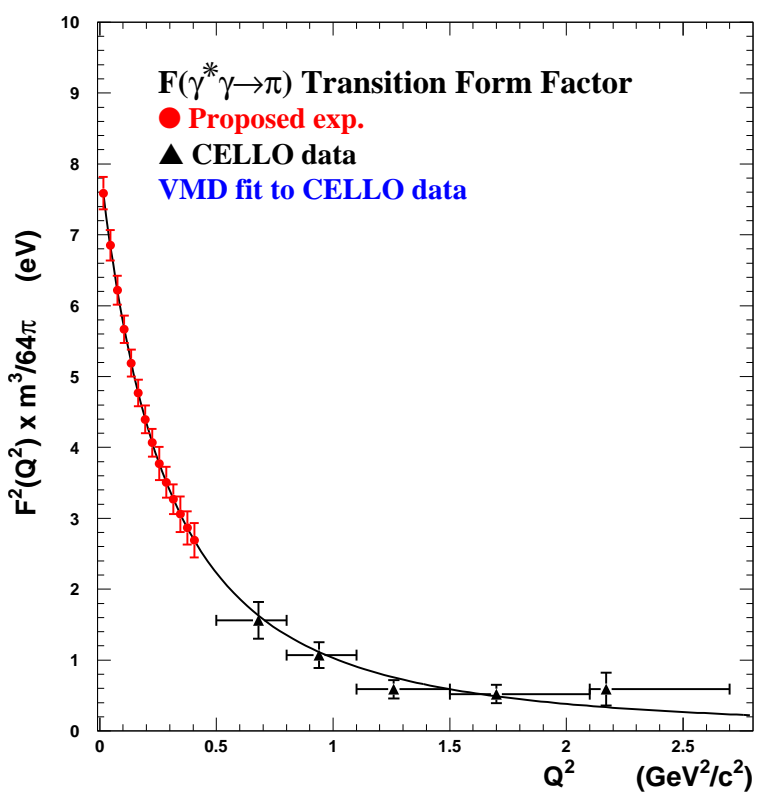

Figure 4. The $\pi^{o}$ transition form factor. The proposed points are projected to the VMD prediction with expected total errors. CELLO data are from reference[12]

\section{Summary}

We have described a comprehensive program to measure the two photon widths and transition form factors of the light pseudoscalar mesons at Jefferson Lab. Precise measurements of these quantities will have a significant impact in the experimental determination of fundamental parameters of QCD. The proposed measurements of the transition form factors at very low $Q^{2}\left(\sim 0.001-0.5 \mathrm{GeV}^{2}\right)$ would provide a first measurement of these important quantities. Physically, these can be approximately thought of as measuring the spatial distribution of the axial anomaly for each of the mesons. In addition, one important reason to better understand the transition form factors of the $\pi^{0}, \eta$ and $\eta^{\prime}$ is that pseudoscalar exchange is the major contribution to the hadronic light-by-light scattering part of the muon anomalous magnetic moment[15].

\section{Acknowledgments}

This work is supported through US Department of Energy grant number DE-FG02-99ER41101. Funds for major experimental equipment were provided under NSF MRI grant number PHY-0079840.

\section{References}

[1] See e.g. Dynamics of the Standard Model, J.F. Donoghue, E. Golowich, and B.R. Holstein, Cambridge University Press (1992).

[2] J.S. Bell and R. Jaciw, Nuovo Cimento 60A, 47 (1969). S.L. Adler, Phys. Rev. 177, 2426 (1969).

[3] D. Haidt et al edit, Review of Particle Physics, Vol 3, (1998).

[4] J. L. Goity, A. M. Bernstein and B. R. Holstein, Phys. Rev. D66, 076014 (2002).

[5] B. Moussallam, Phys. Rev. D51, 4939 (1995). B. Ananthanarayan and B. Moussallam, hep-ph/02052702.

[6] E. Hadjimichael and S. Fallieros, Phys. Rev. C 39, 1438 (1989).

[7] H.W. Atherton et al., Phys. Lett. B 158, 81 (1985).

[8] A. Browman et al., Phys. Rev. Letts. 33, 1400 (1974).

[9] G. Bellettini et al., Il Nuovo Cimento, 66, 243 (1970).

[10] V.I. Kryshkin et al., Sov. Phys. JETP, 30, 1037 (1970).

[11] Phys. Lett. B374, 181 (1996).

[12] H.J. Behrend, et, al., Z. Phys. C 49, 401 (1991).

[13] M. Acciarri et al., Phys. Lett. B418, 399 (1998).

[14] J. Bijnens, A. Bramon and F. Cornet, Phys. Rev. Lett. 61, 1453 (1988).

[15] Andrzej Czarneki and William J. Marciano, Phys. Rev. D 64, 013014 (2001). 